\title{
Computer FOSTAL Chess with CHESSTER The Pawn
}

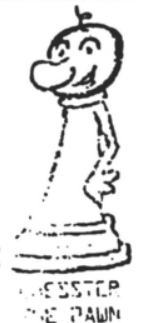

CHESSTER The Pawn was born in 1966- I created him as a cartoon feature for NOST-ALGIA, the of ficial publication of the NOST (Knights Of the Square Table) postal chess club.

In 1977 I purchased my first micro-chess computer; Fidelity's CHESS CHALLENGER III. And Chesster became a bonified dues paying member of NOST, participating in the club's various postal tournaments and Challenge Matches. . Over these 5 years he has graduated to CHESS CHALLENGER 10, then SARGON II. Currently, all of his postal games are being played via the CHESS CHAMPION MARK $\mathrm{v}$.

Chesster's Won, Lost, Draw record to date is:

26 Wins; 26 Losses; 13 Draws.

At present, Chesster is in the midst of a special NOST computer tournament. His opposition: MYCHESS - SFINKS 3.0 - SARGON II - THE GREAT GAME MACHINE (Grunfeld/Morphy/Capablanca) - And two CHESS CHALIENGER ?'s.

On the following page are 2 of Chesster's wins. The first is a quickie over the VOICE CHESS CHALLENGER, from the 1st Annual North American Open Computer Postal Tourney. The second is over human player GILBERT GALLANT, from a NOST postal foursome. . Enjoy!

Would any of you be interested in forming an International Computer Postal Chess Club? . . If so, contact me with your ideas and suggestions!

Lés Roselle

111 Amber St.

Buffalo, NY 14220 


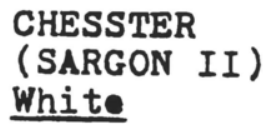

1. $\mathrm{c} 4$

2. $\mathrm{Ne} 3$

3. Nf3

4. 4

5. d3

6. Be2

7. $0-0$

8. Bxf3

9. $\mathrm{Na}_{4}$

10. Bd2

11. $\mathrm{Bg} 5$

12. Bxf6

13. Bg4+

14. Nc3

15. Nd5+

16. Nxf6

17. Nd5

18. Qf3

19. Qf 5
VOICE

CHALIENGER

Black

c5

e 5

Nf6

d6

Bg 4

Bxf 3

Qb6

Qa5

Qa6

Kd?

gxf6

Ke?

Re8

Kd8

Re?

Re8

Be?

Resigns

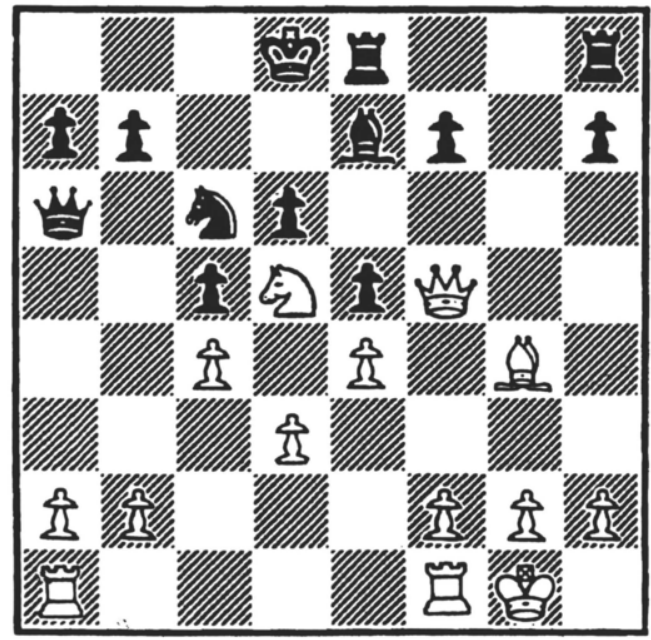

4.

Final position
CHESSTER

(MARK V)

White

1. 4

2. $\mathrm{Ne} 3$

3. exd 5

4. d4

5. Ne3

6. $\mathrm{Bb} 5$

7. $0-0$

8. h3

9. 84

10. Ne 5

11. dxe 5

12. Bxe6+

13. Re 1

14. Bf 4

15. Rxe 5

16. Qe2

17. 85

18. Bh2

19. Qxh5

20. Rae1

21. Qg 4

22. Qa4

23. Qf4

24. Rxe6
G. GALIANT

Black

c6

d5

cxd 5

Nc6

Bg4

e6

Bd6

Bh5

Bg6

Bxe 5

16

bxc6

Qe7

Ixe 5

Ne6

Kd?

Nh5

Bf 5

Bxc2

Bg6

Bf?

Qc5

Rhf8

Resigns

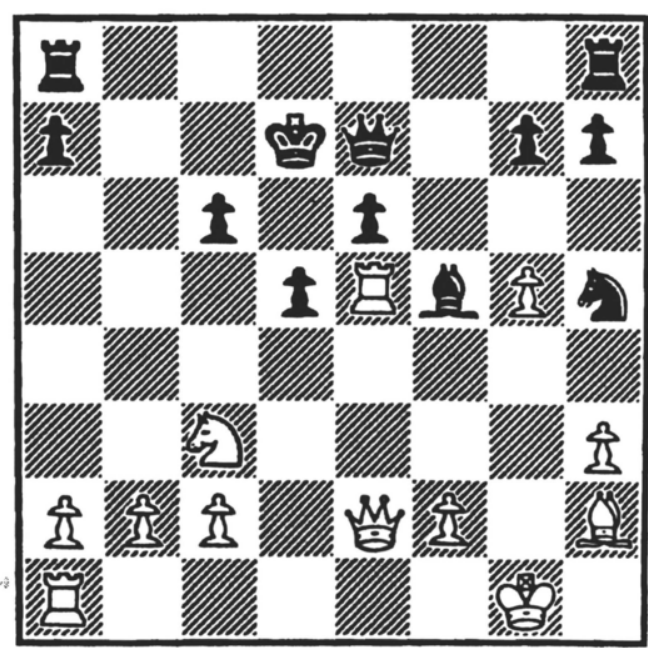

Position after 18. . Bf5 